Revista de Derecho YACHAQ N8 - Diciembre del 2017

Centro de Investigación de los Estudiantes de Derecho (CIED)

Universidad Nacional de San Antonio Abad del Cusco

ISSN: 2707-1197 (en línea)

ISSN: 1817-597X (impreso)

Fecha de recepción: 17/09/17

Fecha de aceptación: 01/10/17

[pp. 21 - 30]

\title{
LAS FUNCIONES DE LAS CORTES SUPREMAS ENTRE UNIFORMIDAD Y JUSTICIA.
}

\section{SUPREME COURTS FUNCTIONS BETWEEN UNIFORMITY AND JUSTICE}

\author{
Michele Taruffo ${ }^{1}$
}

\begin{abstract}
Resumen: La uniformidad en la interpretación y la aplicación del derecho son los principios que constituyen la base de un ordenamiento ideal, uno que va a tener como pilar de su sistema jurídico a las Cortes Supremas y su importante función de representar la finalidad de estos dos principios mencionados. Las técnicas que se utilizan en los diversos ordenamientos para hacer efectivas esas funciones "uniformadoras", irán desde "el stare decisis" en las cortes inglesas, hasta el Acuerdo del Pleno no jurisdiccional del 30 de diciembre del 2011 del Tribunal Supremo en España. Pero estas posturas solo serán aceptadas y defendidas por aquellos ordenamientos apoyados en el concepto de "el universalismo jurídico", uno que recibió muchas críticas por "el particularismo jurídico", él que, polarmente, sostiene que las decisiones deben fundarse solamente sobre las circunstancias particulares del caso concreto sin ninguna referencia a las normas. En este trabajo, se repasará el valor fundamental de la uniformidad en la interpretación y la aplicación del derecho en las funciones encomendadas a las cortes supremas, mencionando las técnicas más utilizadas y las discrepancias que hay entre las concepciones del universalismo y particularismo jurídico.
\end{abstract}

Palabras claves: uniformidad en la interpretación y aplicación del derecho, cortes supremas, universalismo, particularismo, precedentes.

\begin{abstract}
The uniformity in the interpretation and right application are the values that establish the base for an ideal arrangement, one for its legal system has as a pilar the supreme courts and its important function to represent the purpose of these two previeusly mentioned values. The techniques that are used in these various arrangements to ensure these "uniforming" functions range from "el stare decisis" in the english courts to the 2011 December 13th supreme court of Spain non-jurisdictional plenary agreement. But these postures will only be defended for those arrangements supported in the "legal universalism" concept wich received a lot of critics from the "legal particularism" which holds polarly that decisions must be founded only over particular circumstances from the especific case witouth any reference to the norms. This work will review the fundamental value of uniformity in the interpretation and application of the law in the entrusted functions to the supreme courts mentioning the most used techniques and the discrepacies between the legal universalism and the legal particularism.
\end{abstract}

Key Words: Uniformity in the interpretation and application of the law, supreme courts, universalism, particularism, precedents.

\footnotetext{
${ }^{1}$ Jurista italiano, especialista en Derecho Procesal Comparado y Derecho Procesal Civil. Profesor de la Università degli Studi di Pavia, Ha sido profesor visitante,en forma habitual de varias universidades europeas, estadounidenses y latinoamericanas Catedrático de Cultura Jurídica, Girona.

2 A propósito, se puede observar en passant que este argumento, muy difundido en muchos ordenamientos jurpidicos que apunan a utilizr el precedente, es sie estomado a lamletra- filosificamente inconsiente. Eso viola en efecto las leyes planteadas por Leibniz en la identidad de los discernibles, según, el cual dos hechos iguales no existen, y si dos hechos se dice que son iguales esos en realidad es un solo hecho. Es común que suceda, además, que los juristas no son sofisticados filósofo.tanuffo@iusspavia.it
} 


\section{Las Funciones De Las Cortes Supremas ENTRE UNIFORMIDAD Y JUSTICIA. ${ }^{1}$}

\section{Michele Taruffo}

Jurista italiano, especialista en Derecho

Procesal Comparado y

Derecho Procesal Civil.

Profesor de la Università degli Studi di Pavia, Ha sido profesor visitante, en forma habitual de varias universidades europeas, estadounidenses

y latinoamericanas

Catedrático de Cultura

Jurídica, Girona.
SUMARIO: 1.- El Valor de la Uniformidad, 2.- Las Funciones de las Cortes Supremas, 3.- Universalismo y Particularismo, 4.Conclusiones

\section{1.- EI Valor de la Uniformidad}

L a uniformidad en la interpretación y la aplicación del derecho constituye desde hace mucho tiempo un valor fundamental (se podría decir incluso: un objeto de deseo) presente en prácticamente todos los ordenamientos jurídicos, que buscan en varios modos - como se verá más adelante- de concretizarlo en la más amplia medida posible.

Las justificaciones que están a la base de esta aspiración general son varias y no se podría examinar en este documento en modo completo . Para indicar solamente aquellas a las cuales se hace mayor referencia se puede recordar, sobre todo, la exigencia de la certeza del derecho, dado que una jurisprudencia uniforme evita la incerteza en la interpretación del derecho y la consecuencia variedad o cambiamientos de las decisiones judiciales. Se hace mención además a la garantía de la igualdad de los ciudadanos de frente a la ley, en base al principio de la decisión vinculante (stare decisis) típico de los ordenamientos jurídicos angloamericanos, según el cual, casos iguales deben ser decididos en modo igual ${ }^{2}$

\footnotetext{
1 Para mayor información, sobre el argumento: TARUFFO, Giurisprudenza, in Enc.Treccani Sc.Soc., vol.IV, Roma 1994, p.357. V. inoltre BANKOWSKIMACCORMICK-MORAWSKI-RUIZ MIGUEL, Rationales for Precedent, in Interpreting Precedents. A Comparative Study, ed.by D.N.MacCormick and R.S.Summers, Aldershot-Brookfield-Singapore-Sydney 1997, p. 481 ss

2 A propósito se puede observar en passant que este argumento, muy difundido en muchos ordenamientos jurpidicos que apunan a utilizr el precedente, es - sie estomado a lamletra- filosificamente inconsiente. Eso viola en efecto las leyes planteadas por Leibniz en la identidad de los discernibles, según, el cual dos hechos iguales no existen, y si dos hechos se dice que son iguales esos en realidad es un solo hecho. Es común que suceda, además, que los juristas no son sofisticados filósofos.
} 
Se da además mucha importancia, a menudo, a la predecibilidad de las decisiones futuras, sobre la cual las partes deberían poder hacer referencia, sobre el hecho, que los jueces futuros decidirán del mismo modo como lo hicieron los anteriores. La predecibilidad puede desenvolver, además, una función económica, dado que si las decisiones judiciales son predecibles, se puede evitar recurrir al juez. En fin, una jurisprudencia constante en la interpretación y la aplicación de las normas, se pueden conocer más fácilmente y en consecuencia orientar en modo más eficaz los comportamientos de los socios.

Es fácil observar que estas justificaciones no se contradicen entre sí, en todo caso, convergen hacia la necesidad que la interpretación y la aplicación de derecho sean lo más uniforme posible y constante. Sobre esta base, y lamentablemente las respectivas diferencias de formulaciones, se vislumbra una imagen ideal del ordenamiento jurídico según, la cual, debería ser completo y sincronizado coherentemente, estático y uniforme, y debería también asegurarse que todas las controversias que aparecen en el interno, encuentren un solución estable y unitaria.

Queda en segundo plano, en cambio, o a los márgenes del horizonte conceptual entre las cuales se coloca esta imagen, la dimensión del ordenamiento que se podría llamar dinámica y diacrónica, porque esa supone que en el sistema haya incertezas y variaciones, diferencias y mutamientos. La existencia de esta dimensión es indudable e inevitable, como se verá mejor, a continuación, pero esta posición tiende a verse en forma negativa, como una excepción que -como tal- debería ser el más posible reducida y circunscrita. En consecuencia, se admite y, -no podría ser diversamente- la variación al interno de la jurisprudencia, pero se tiende a considerarlo posible solo cuando existen razones particularmente relevantes, que comúnmente se indican en términos vagos con referencia a cambiamientos sociales, morales y económicos. No es una casualidad que a veces se tienda a conciliar las opuestas exigencias de la predecibilidad y del cambiamiento de la jurisprudencia, como sucede por ejemplo en la prospective overruling de la Corte Suprema de los Estados Unidos ${ }^{3}$.

\section{2.- Las Funciones de las Cortes Supremas}

La ejecución de la finalidad representada por la uniformidad de la interpretación y aplicación del derecho viene, generalmente, confiada, sobre todo, a las cortes supremas ${ }^{4}$. Se trata en todo caso de un aspecto importante -tal vez el más importante- de eso que llamaría el mito de las cortes supremas: esa se coloca al centro del sistema jurídico y al ápice de la estructura judiciaria, y se tiende a pensar que en eso se concrete el aspecto más relevante de la administración de justicia. De aquí la consecuencia que se espera de estas cortes, la función fundamental de asegurarse el valor representado por el valor de la uniformidad de la jurisprudencia.

Se trata ciertamente de cosas importantes: en cuanto corresponde a Italia, basta hacer referencia a Calamandrei, en la Casación Civil ${ }^{5}$, sobre la unificación de la casación realizada en $1923^{6}$, y al artículo 65 de la Ley del 41 sobre

\footnotetext{
Para más información: TARUFFO, Giurisprudenza, cit., p.561.

4 Sobre las principales funciones que vienen atribuidas a estas cortes, ver: TARUFFO, Le funzioni delle Corti Supreme. Cenni generali, in Ann.dir.comp.e st.leg. 2011, p.11 ss.. Di una funzione jurisprudentielle et normative della Cour de Cassation francese parla ad es. CADIET, Le rôle institutionnel et politique de la Cour de Cassation en France: tradition, transition, mutation?, ivi, p.191 ss.. Cfr.inoltre i saggi raccolti nel volume The Role of the Supreme Courts at the National and International Level, ed by P.Yessiou-Faltsi, Thessaloniki 1998.

5 Cfr. CALAMANDREI, La Cassazione civile.II.Disegno generale dell'istituto (1920), in ID., Opere Giuridiche, vol.VII, Napoli 1976, p.57 ss.

6 Cfr. TARUFFO, Calamandrei e l'unificazione della Cassazione, in ID., Il vertice ambiguo. Saggi sulla Cassazione civile, Bologna 1991, p.51 ss., e da ultimo RICCI, Il giudizio civile di Cassazione, Torino 2013, p.23 ss
} 
el ordenamiento judicial, hasta ahora en vigencia, donde se lee que la corte "asegura la exacta observancia y la uniforme interpretación de la ley", además de garantizar "la unidad del derecho objetivo nacional"7.

Es aun interesante observar que los diversos ordenamientos emplean varias técnicas para disciplinar las modalidades con las cuales deben actuar las funciones "uniformadoras" de las respectivas cortes supremas. La más difundida de estas técnicas es sin duda constituida por el stare decis, ósea la referencia a una decisión anterior, y esencialmente a la ratio decidendi que justifica jurídicamente el éxito, de la capacidad de influenciar ${ }^{8}$ la decisión de un caso sucesivo idéntico o análogo ${ }^{9}$. Nata principalmente en las cortes inglesas y norteamericanas, la praxis del precedente se ha difundido en muchísimos ordenamientos incluso del civil law ${ }^{10}$. En algunos casos, más bien, la referencia al precedente se ha convertido en una especie de mantra que viene recitado repetidamente, hasta constituir una suerte de obsesión que influye también el legislador procesal.

Es significativo al respecto el ejemplo italiano, dado que el reciente legislador está tentando en todos modos, sin lograrlo (dada la escaza calidad técnica de las normas que plantea) de hacer que en Italia se instaure una seria praxis del precedente, naturalmente bajo la iluminada guía de la Corte de Casación. Basta recordar el ya tristemente famoso "filtro" de los recursos en casación previsto en el artículo 360 bis n.1) del cod.proc.civ., introducido en el 2009, según el cual, sería inadmisible el recurso si la sentencia impugnada este conforme a la jurisprudencia de la Casación (y el recurso no ofrezca elementos que induzcan a confirmar o a cambiar tal jurisprudencia) ${ }^{11}$. Aparte de las numerosas críticas de carácter técnico, sobre la cual no vale la pena insistir, se puede destacar que el legislador italiano, ignora en manera total que cosa es el precedente: en efecto viene descuidada la naturaleza fundamental del verdadero precedente, ósea - como se ha dicho- la analogía entre los hechos de los dos casos y, si considera como precedente cualquier afirmación abstracta de la Corte de Casación sobre una quaestio juris en cualquier modo referible al caso en análisis ${ }^{12}$. La consecuencia es que en la mayor parte de casos el "precedente" de la Corte de Casación no es la sentencian en su integridad, es en cambio una máxima de pocas reglas que enuncia una regla en términos generales y abstractos ${ }^{13}$.

Una técnica muy diversa consiste en configurar la posibilidad que la corte suprema emita pronunciamientos dotados de eficacia ultra partes, ósea de efectos vinculantes capaces de determinar directamente las decisiones de casos sucesivos. El ejemplo más notable ha sido en el pasado las directivas de la corte suprema de la URSS, pero no se olvida que esta técnica esta todavía presente en la casación cubana, que sigue el modelo soviético. En Rusia, además,

\footnotetext{
7 Sobre el particular: TARUFFO, La Corte di Cassazione e la legge, in Id., Il vertice ambiguo, cit., p.59 ss., 70 ss.. V. inoltre RICCI, Il giudizio civile, cit., p.32 ss.

8 Uso aquí deliberadamente un término atécnico y genérico, con la finalidad de tomar posición sobre la vexata quaestio, que conoce soluciones diversas, incluso en función de diferentes contestos de ordenamientos jurídicos relativos a si el precedente tenga una eficacia vinculante o solo persuasiva. En este momento no nos corresponde y no es posible afrontar el tema. Queda en todo caso, el ensayo de PECZENIK, The Binding Force of Precedent, in Interpreting Precedents, cit., p.461 ss.

9 No repito la referencia ya hecha a las leyes de Leibniz. Una correcta aplicasión necesitaria de todos modos de precisar la aplicación de la ratio decidendi que ha fundado la decision sobre el caso precedente, al caso sucesivo, requiere que el segundo juez realiza una suficiente analogia entre el hecho del caso que debe decidir y el hecho que ha sido objeto de decisión precedente. Sobre el particular ver: SERIO, La rilevanza del fatto nella struttura del precedente giudiziario inglese, in Casistica e giurisprudenza, a cura di L.Vacca, Milano 2014, p.91 ss.

${ }_{10}$ La literatura sobre el precedente es actualmente inmensa, al punto que cualquier indicación bibliográfica sería incompleta e che qualunque indicazione bibliografica sarebbe incompleta desconfiable. Se vea, en todo caso, un util análisis comparativo de ensayos recogidos en Interpreting Precedents, cit.

11 Sobre este argumento, se ha creado un interesante debate: v RICCI, Il giudizio civile, cit., p.209 ss.; POLI, Le modifiche relative al giudizio di cassazione, in PUNZI, Il processo civile. Sistema e problematiche. Le riforme del quadriennio 2010-2013, Torino 2013, p.193 ss.

12 TARUFFO, Precedente e giurisprudenza, in Riv.Trim.Dir.Proc.Civ., 2007, p.709 ss.

13 TARUFFO, op.ult.cit., p.712 ss.
} 
esta práctica, sigue con los decretos -verdaderos y propios actos normativos- que son emanados por el plenum de la corte suprema con eficacia vinculante para todos los jueces que se encuentran en la posibilidad de aplicar las mismas normas o a juzgar sobre casos análogos, es aplicable, incluso para todos los órganos administrativos. El mismo sistema se aplica a los decretos de la corte suprema comercial, y también a las decisiones que el presídium de esta corte emana en sede de revisión de casos concretos ${ }^{14}$.

Una técnica análoga, muy interesante, y en cierto sentido extrema, es aquella que hace bisagra sobre las súmulas vinculantes del sistema brasiliano. La formulación de las súmulas sobresale a una práctica consolidada en ese ordenamiento: ellos en el pasado no tenían eficacia vinculante, mientras que ahora, tienen esa eficacia, después de una reforma constitucional del $2004^{15}$. Se trata de enunciaciones que son formuladas por el Supremo Tribunal Federal después de una reunión de sus miembros y una votación (con una mayoría de los dos tercios). Ellos tienen la función de resolver una contradicción que se haya verificado en la jurisprudencia de las cortes inferiores. Vale la pena observar que la súmula no deriva de la decisión de un caso concreto, porque se trata de una enunciación interpretativa formulada en términos generales. En consecuencia, no se hace referencia a los hechos que están en la cuestión jurídica afrontada, en consecuencia, no puede ser considerado como un precedente en sentido propio, es solo una decisión que da a conocer la elección entre dos opciones interpretativas relativas a normas generales y abstractas ${ }^{16}$. La función, es evidente, que es, la eliminación de incertezas y conflictos en el ámbito de la jurisprudencia, asegurándose la uniformidad. A este fin se prevé que tenga eficacia vinculante para todos los jueces y también para todos los órganos públicos del Estado Federal.

Algo similar, aunque no comparable por la importancia y la dimensión a la experiencia brasiliana, se ha verificado hace poco, en España: con un Acuerdo del Pleno no jurisdiccional del 30 de diciembre del 2011 el Tribunal Supremo español ha indicado, con un acto, que no era la decisión sobre un recurso, pero si una declaración extrajudicial, los propios orientamientos relativos a la interpretación de una norma de gran importancia sobre la característica de la admisibilidad del recurso al mismo Tribunal, ósea del artículo 477 n. 3 de la Ley de Enjuiciamiento Civil. Se trata de la norma que subordina la admisibilidad del recurso a la presencia de un interés casacional, y con la finalidad de clarificar como debe ser interpretada esta cláusula, de significado incierto, que el Tribunal haya emanado esa declaración ${ }^{17}$.

A veces, por tanto, se hace referencia más o menos apropiado a la eficacia del precedente en sentido propio; otras veces, se cae en el error entorno a eso que constituye o no constituye un precedente, en otras veces, incluso, se abandona la referencia a la técnica del precedente para recurrir a pronunciamientos o a disposiciones que no son precedentes, dado que no vienen pronunciados en ocasión de las decisiones de casos concretos y tienen más bien la naturaleza de actos normativos, a pesar de no ser de fuente legislativa. Sin embargo, aparte de la variedad de estas técnicas y de las diferencias que corresponden los efectos que puedan producir, una orientación constante está en el sentido de individualizar en las cortes supremas los órganos a quien se confía la tarea de asegurar la uniformidad de

${ }^{14}$ Cfr. RUDOVKAS, Precedente giudiziario come fonte del diritto nell'ordinamento giuridico della Federazione Russa, in Casistica e giurisprudenza,cit., p.129 ss.

15 Cfr. HIGASHIYAMA, Teoria do Direito Sumular, in Rev.de processo 2011, p.74 ss.; BAHIA, As Súmulas Vinculantes e a Nova Escola da Exegese, ivi 2012 , p.362 ss.

${ }^{16}$ Cfr. HIGASHIYAMA, Teoria, cit, p.87,99

${ }^{17}$ Cfr. DE LA OLIVA SANTOS-DÍEZ-PICAZO GIMÉNEZ-VEGAS TORRES, Curso de derecho procesal civil.II.Parte especial, Madrid 2012, p.282 ss. 
las interpretaciones y las aplicaciones del derecho. En eso no haya de sorprendente, tratándose de corte colocadas a la cumbre de la pirámide judicial. De este modo se exalta la característica de colocar en la cumbre de los órganos, en cuanto se prevé que la corte pueda imponer -en un modo o en el otro-las propias decisiones interpretativas y aplicativas a todos los jueces colocados en las posiciones inferiores de la pirámide. Es decir, corresponde al modelo clásico de la burocracia concentrada, sobre el cual, el poder aumenta y se concentra cuando de la parte más baja se sube hasta la parte más alta, y se convierte en el máximo cuando se alcanza la cumbre. Naturalmente este modelo implica que el poder sea inferior y desconcentrado cada vez que se desciende de la cumbre hasta la base, ósea al juez de primera instancia.

Sobre otro punto de vista, se advierte que sobre este modelo de ordenamiento la corte suprema termina siendo más importante que el legislador: a ellos corresponde la función de producir normar generales y abstractas, pero es la corte suprema que establece el significado que a la normas debe ser atribuido y, que en consecuencia debe ser tendencialmente aplicado por todos los jueces inferiores y, en consecuencia a todos los sujetos del ordenamiento, sin contar que a menudo -como se ha visto en los ejemplos citados anteriormente- es la misma corte suprema a producir normas generales y abstractas con 1 finalidad de vincular la interpretación que puede ser dado por otros órganos.

\section{3.- Universalismo y Particularismo}

La concepción que sumariamente se ha expuesto constituye una versión específica, pero difusa, de lo que los filósofos llaman "universalismo jurídico"18, fundado esencialmente sobre la idea que existen reglas generales destinadas a ser aplicables en modo uniforme de parte de los juristas, y que las decisiones relativas se justifiquen solo si el caso particular que es objeto de decisión pueda ser subsumido en una norma general que debe aplicarse en el mismo modo en todos los casos iguales o similares. En esta versión, el elemento característico está representado del hecho que -como se ha visto- se ha atribuye a la corte suprema la función de estabilizar cuales son las reglas generales que deben ser atribuidas a cada caso singular.

El universalismo jurídico es objeto de varias críticas, fundadas sobretodo en la consideración -que ya constituye un lugar común entre filósofos del derecho- che la decisión no es más el fruto de la aplicación mecánica de normas legales, y que en todo caso, la aplicación de la norma no puede darse sino haciendo referencia a la complejidad del caso particular sobre la cual se da la decisión. Los orientamientos que en varios modos siguen están perspectiva se conocen comúnmente como "particularismo jurídico"19, y hacen referencia en particular al razonamiento al razonamiento con el cual se formula las decisiones judiciales ${ }^{20}$.

El particularismo se presenta en diversas formas, algunas de las cuales son ya radicales y llegan a sostener que las decisiones deben fundarse solamente sobre las circunstancia particulares del caso concreto sin ninguna referencia a las normas. No es el caso de tomar en consideración esta tesis ${ }^{21}$. Se puede aún observar que también se siguen

\footnotetext{
18 BOUVIER, Particularismo y derecho. Un abordaje postpositivista en el ámbito práctico, Madrid-Barcelona-Buenos Aires 2012 , p. 19 ss., 21 ss., 38 ss.. Cfr. inoltre GROSSI, Universalismo e particolarismo nel diritto, Napoli 2011; SACCO, Il diritto tra uniformazione e particolarismi, Napoli 2011; PALAZZO, Il diritto penale tra universalismo e particolarismo, Napoli 2011.

19 Para un amplio análisis: cfr. Bouvier, Particularismo, cit., p.58 ss., 175 ss., 309 ss., 349 ss., e gli altri scritti citati nella n.14. Cfr. inoltre Taruffo, La semplice verità. Il giudice e la costruzione dei fatti, Bari 2009, p.200 ss., anche per altri riferimenti

${ }^{20}$ Cfr. Los ensayos recogidos en el volumen The Universal and the Particular in Legal Reasoning, ed.by Z.Bankowski and J.MacLean, Aldershot 2006.

${ }^{21}$ PAVLAKOS, Two Conceptions of Universalism, in The Universal, cit., p.163 ss.; TARUFFO, La semplice verità, cit., p.200.
} 
versiones moderadas del particularismo, como aquella que sostiene que siempre es necesario la referencia a las reglas generales incluso cuando las decisiones se concentren sobre particularidades del caso específico ${ }^{22}$ es siempre la importancia que se viene dando a las particularidades que determina la configuración de las reglas que constituyen el criterio de decisión. Derivan algunas consideraciones tal vez no privadas de importancia en el discurso en el cual se desarrollan.

Sobre todo se precisa que la decisión judicial no consiste en la mera enunciación de reglas (o de máximas) presentadas como la única interpretación abstractamente correctas, uniformes y vinculantes, de disposiciones normativas, puesto que -como ya uniformemente se reconoce- es el intérprete a escoger y a determinar su significado ${ }^{23}$. Por otra parte, se necesita reconocer que se pueden dar buenas razones para decidir casos similares en modos diversos: puesto que no existen dos hechos iguales ${ }^{24}$, es más bien sobre las diferencias, ósea sobre los particulars, que debe fundarse la interpretación de la norma que debe ser aplicada al caso específico. Parece evidente que cuando una norma viene interpretada para derivar la regla del juicio a aplicarse a un caso concreto, como sucede en el proceso, es la referencia a los hechos del caso a guiar la interpretación de la norma ${ }^{25}$. En caso contrario, ósea si la norma no viene interpretada con referencia a esos hechos, la consecuencia es que la norma no es aplicable al caso ${ }^{26}$. Análogamente, como ya se ha señalado ${ }^{27}$, es esencialmente analizando los hechos y estableciendo una suficiente analogía entre los hechos del caso precedente y los hechos del caso sucesivo que el juez de este segundo decide acerca de la aplicación de la ratio decidendi que constituye el precedente.

En definitiva, es el hecho que determina la interpretación de la regla del derecho que a ese hecho debe ser aplicado. No es de sorprender que es la relación de la norma con el hecho la que constituye uno de los problemas fundamentales de la teoría del derecho ${ }^{28}$ y del derecho procesal ${ }^{29}$. En consecuencia, son las peculiaridades de los hechos de varios casos que pueden llevar a diversas interpretaciones de la misma regla y, en consecuencia a no aplicarlas en casos aparentemente similares o a aplicarlas en casos aparentemente diversos. Este discurso no puede ser aquí tratado como debería ser, pero cualquier ejemplo puede ser útil para clarificar el significado.

Un primer ejemplo puede estar constituido por una situación que desde un tiempo acá es objeto de particular atención de parte de los constitucionalistas y de los filósofos del derecho: se trata de c.d. balance o (ponderación) entre principios, que ocurre cuando se trata de resolver antinomias entre diversos principios constitucionales. En tal caso son las particularidades del caso que debe ser decididas para determinar la elección favorable a la aplicación de un principio en lugar que la aplicación de otro principio ${ }^{30}$.

\footnotetext{
22 Ver Pavlakos, Two Conceptions of Universalism, in The Universal, cit., p.163 ss.; Taruffo, La semplice verità, cit., p.200.

23 Gentili, Il diritto come discorso, Milano 2013, p. 8 ss., 15 ss.

24 Sobre el punto MACCORMICK, Particulars, cit., p.5, e v. supra, n. 2.

${ }^{25}$ TARUFFO, IL FATTO E L'INTERPRETAZIONE, IN LA FABBRICA DELLE INTERPRETAZIONI, A CURA DI B.BISCOTTI, P.BORSELLINO, V.POCAR E D.PULITANÒ, MILANO 2012, P.123 SS

26 TARUFFO, LA SEMPLICE VERITÀ, CIT., P.199.

${ }^{27}$ V. SUPRA, N.9 E EL TEXTO.

${ }^{28}$ ENGISCH, LOGISCHE STUDIEN ZUR GESETZESANWENDUNG, 2 AUFL., HEIDELBERG 1960

29 NITSCH, IL GIUDICE E LA LEGGE. CONSOLIDAMENTO E CRISI DI UN PARADIGMA NELLA CULTURA GIURIDICA ITALIANA DEL PRIMO NOVECENTO, MILANO 2012

${ }_{30}$ LUZZATI, Il giurista che cambia e non cambia, in Dir.pubbl., 2013, 2, p. 432
} 
Un segundo ejemplo, que parece significativo en varios países de América Latina, respecta la situación en el cual existen poblaciones indígenas a las cuales vienen aplicadas particulares condiciones de autonomía, hasta la creación de especiales condiciones jurisdiccionales. En estos casos parece evidente el reconocimiento explícito de diferencias subjetivas relevantes, de frente a las cuales parece difícil, si no imposible, predicar la absoluta uniformidad de la interpretación y de la aplicación de las normas en el ordenamiento jurídico del Estado.

Otro ejemplo se individualiza cuando se reconoce que las características étnico-culturales del autor de un delito son significativas a los fines de la decisión que debe ser tomada en relación a este sujeto que ha cometido el delito, por ejemplo en términos de punibilidad o de determinación de la pena ${ }^{31}$. En estos casos, en efecto la decisión tiene en cuenta las específicas características culturales del sujeto, derivadas de su pertenencia a un particular grupo étnico.

Estos ejemplos, pero además muchos otros más se podrían realizar, mostrando que son numerosas situaciones de las cuales surgen aspectos relevantes que inducen al juez a emplear diversos argumentos jurídicos, por ejemplo, haciendo referencia de vez en cuando, a diferentes principios generales o constitucionales ${ }^{32}$, y también a invocar varias razones meta jurídicas, inspiradas en diferentes valores sociales o morales que orientan la heterointegración de la norma. Parece, en consecuencia evidente la falta de fundamento de tesis como aquella de la one right answer anunciada en su tiempo por Dworkin, según el cual un juez "hercúleo" podría siempre encontrar la verdadera y única interpretación correcta de cada norma ${ }^{33}$. Por otro lado, i c.d hard cases son a menudo hard en los hechos, y es esta misma complejidad de los hechos a hacer difícil la interpretación y la aplicación de la norma a la que se hace referencia.

Entonces necesita reconocerse que la interpretación de la norma dirigida a su aplicación como regla de juicio en un caso concreto tiene una inevitable y muy relevante aspecto particular, debiéndose fundar sobre una completa y analítica consideración de los particulars del caso $^{34}$ : es sobre los particulars, por tanto, que se va modelando la interpretación de la norma.

Naturalmente eso conlleva un posterior problema relevante: porque las circunstancias que constituyen los hechos del caso son infinitas y, son también infinitas las posibilidades discrecionales ${ }^{35}$, se trata de determinar cuáles son los particulars que se consideran significativos y relevantes para la interpretación y la aplicación de la norma a la cual se hace referencia ${ }^{36}$. Al respecto no existen soluciones simples que puedan valer automáticamente para cualquier decisión. Incluso la referencia al caso definitivo en términos generales de la norma sirve solo -por así decirlo- a dar inicio al análisis de los hechos, dando un paradigma inicial, que en realidad no es más una hipótesis provisional, de relevancia jurídica ${ }^{37}$. Es pero, moviéndose de esta hipótesis y, eventualmente formulando otras que se den progresivamente las hipótesis más adecuadas a las circunstancias del caso concreto que el juez, a través de

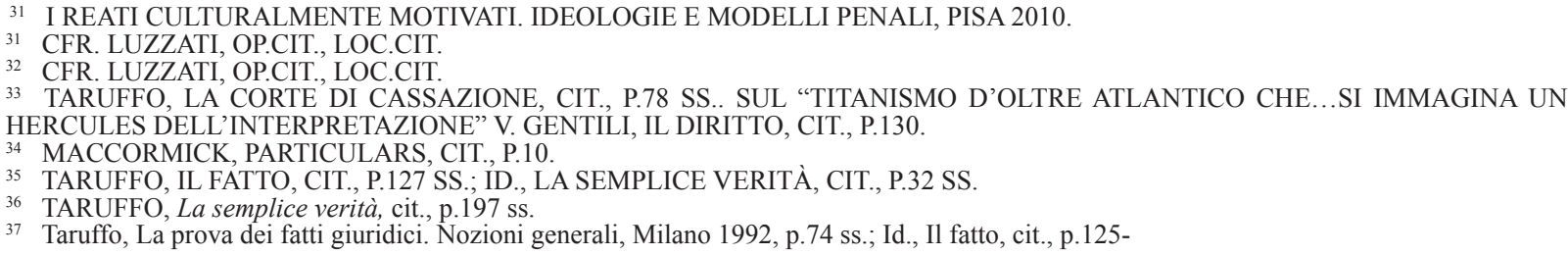


la hermenéutica, llegue a establecer cuáles son las circunstancias relevantes del caso. Porque, de otro modo, en este procedimiento de trial and error o -si se prefiere- de abducción interpretativa, no hay nada de mecánico o predeterminado, es una actividad eminentemente creativa aquella que lleva al juez a atribuir mayor relevancia a determinados particulars del caso y, a modelar sobre esos la interpretación de la norma que piensa aplicar ${ }^{38}$

Si se toma en cuenta estas consideraciones, pero también otras se podrían invocar a favor del particularismo, como sobre todo su mayor adherencia a lo que sucede realmente en la administración de justicia, la versión del universalismo jurídico de la cual se ha hablado anteriormente aparece caracterizada, como ya se ha señalado, como una concepción fuertemente burocrática y sustancialmente autoritaria. Eso parece hacer evidente en el momento en el que se consagra un modelo piramidal en el cual es solo a la cumbre a "decir el derecho", en modo vinculante para todos e once and forever. Por demás, en este modo el derecho viene "dicho" con fórmulas y un alto grado de abstracción, sin considerar, una cosa obvia, ósea que mientras se suba más en alto en la escala de abstracción más se pierde de vista, lo concreto, la realidad. Resulta, una imagen parcial, unilateral y deformada de la administración de justicia, casi que esa se desarrolla solo en el nivel de las cortes supremas mientras que las decisiones de las cortes inferiores y, sobre todo, la de los jueces de primera instancia, esas mismas que se ocupan de la comprobación de los hechos y de la aplicación de las normas en los casos articulares, fuesen irrelevantes.

En efecto, se observa que si se toma en consideración el particularismo, también en sus versiones moderadas y razonables, se constata que esas no sirven para cortes que, como la Corte de Casación italiana y las otras en las cuales se ha indicado el precedente, se expongan casi exclusivamente a través de enunciaciones generales que no hacen ninguna referencia a los hechos que todavía son objeto de decisión, o incluso no se ocupan para nada de las situaciones concretas a las cuales las normas vienen aplicadas. El particularismo tiene en cambio un aspecto importante con referencia a las cortes que interpretan normas con la finalidad de hacer aplicaciones a casos particulares, ósea principalmente cuando se analizan las modalidades de decisiones que puedan considerarse típicas de los jueces de mérito.

\section{4.- Conclusiones}

Si las consideraciones que preceden tienen un sentido, nos encontramos de frente a una tensión -si no propio a una contradicción- entre dos valores o paradigmas divergentes ${ }^{39}$ : de una lado está la versión rigurosa de la tesis universalista, según el cual el juez estaría vinculado a decidir en modo uniforme, aplicando deductivamente una regla general sin tener en cuenta los hechos del caso, si no para construir en modo tal de hacerle corresponder al hecho abstracto definido por una norma; del otro lado está la perspectiva particularista según la cual, en su versión extrema, el juez debería decidir teniendo en cuenta solamente los hechos particulares del caso específico, sin tomar en consideración alguna regla general.

Los dos paradigmas mencionados parecen además presuponer dos ideas diversas de la justicia. El universalismo se refiere a la justicia como correcta interpretación de disposiciones normativas, ósea a aquellas que se podrían llamar "justicia de las normas", de individualizar en modo autoritativo, posiblemente vinculante, con formulaciones

\footnotetext{
38 Cfr. TARUFFO, La semplice verità, cit., p.203.

39 De una tensión análoga habla: Bell, The Institutional Constraints on Particularism, in The Universal, cit.,
} 
generales, de parte de los órganos de la cumbre del sistema judiciario. El particularismo, en cambio, se refiere a aquella que se podría definir como "justicia de las decisiones" referidas a los singulares casos concretos, ósea a la justicia sustancial de eso que el juez dice refiriendo las normas a las situaciones reales y efectivas, visto en todos los aspectos relevantes, que son objeto de decisión. Si el universalismo alude a una "justicia de las normas abstractas", el particularismo se refiere a la "justicia del caso concreto".

Desde el punto de vista teórico la versión moderada del particularismo no excluye pero, como se ha visto precedentemente, que puedan o deba haber situaciones intermedias, en las cuales la atención necesaria para todos los particulars relevantes se conjuga con la individualización de reglas tendencialmente universales, capaces de ser aplicadas también en otros $\operatorname{casos}^{40}$. No se excluye en consecuencia, admitiendo que se razone teniendo en cuenta las características irrepetibles de los singulares casos concretos, que se espere de la corte y, en particular de las cortes supremas, la tarea de hacer relativamente previsible y controlable la interpretación de las normas ${ }^{41}$.

Bajo el perfil que acá nos interesa mayormente se puede decir que las decisiones judiciales se colocan en el espacio intermedio entre dos extremos constituidos por el universalismo abstracto y por el particularismo absoluto. Teniendo en cuenta del hecho que no existe alguna "regla de oro" que determine un ideal punto de equilibrio entre los dos paradigmas, se puede sólo observar que cada decisión particular puede colocarse en cualquier punto del continuum (en Italia se refiere a todo aquello que no sufre interrupción de continuidad) así determinado, en consecuencia podrá acercarse más al paradigma universalista o al paradigma particularista, o realizarse una oportuna combinación entre los dos paradigmas. A propósito se puede todavía realizar una distinción non privada de importancia: si se considera la jurisprudencia que opera produciendo máximas o súmulas como sucede en las cortes supremas a las cuales se ha hecho referencia anteriormente, entonces parece evidente que se colocan en un punto bastante cercano al extremo universalista, y tal vez -en los ejemplos de peores formalismos- viene incluso a coincidir con aquellos. En cambio, si se considera las decisiones producidas por las cortes inferiores y, en particular de los jueces de primer grado, entonces nos encontramos en un punto más cercano al paradigma particularista, al menos en la medida en la cual estos jueces individualizan y aseguran todas las circunstancias relevantes de los singulares casos concretos. Se podría, por otro lado imaginar una situación ideal en el cual la corte suprema, como tal depositaria e intérprete del paradigma universalista, sea todavía sensible a la exigencia de justicia que nace de las situaciones concretas que son objeto de decisión y, formula sus propias interpretaciones -mientras son destinadas a ser aplicadas en modo uniforme- teniendo en cuenta el máximo posible de las circunstancias relevantes de estas situaciones, sobre todo, en el rápido variar de las condiciones económicas, sociales y políticas, en las cuales surgen las controversias. Recíprocamente, los jueces de los singulares casos concretos no deben adoptar conductas de particularismo absoluto y deberían en cambio hacer referencia a las indicaciones interpretativas que provienen de las cortes supremas, al menos hasta que estas den interpretaciones de reglas de juicio capaces de realizar la justicia en el caso concreto.

Entre ambas cuestiones paradigmáticas de decisiones, y los relativos conceptos de justicia, pueden -y tal vez debanconvivir en el mismo ordenamiento jurídico. Eso significa que queda por establecer, pero no es un problema que debamos afrontarlo en esta sede, es si entre ambos modelos corresponden -cada uno a su modo y por razones diversas- a una concepción global y compleja de la "administración de justicia" en sentido propio. Se pude todavía

\footnotetext{
40 MacCormick e di Walker citati nella n.22.

41 Luzzati, op.cit., p.437, con riferimento specifico alla ponderazione dei principi.
} 
admitir que sea justicia formal aquella en la cual todos son tratados en modo igual en un sistema de reglas generales, $\mathrm{y}$, sea en cambio justicia sustancial aquella que viene hecha teniendo en cuenta los valores y los objetivos del derecho y reportándolos a las circunstancias de los casos particulares ${ }^{42}$. Dependerá entonces de las decisiones políticas formuladas en cada caso concreto del sistema judicial si la prevalencia viene atribuida a la justicia formal universalista mejor que a la justicia sustancial particularista, o si se realiza la combinación ideal, de la cual se ha señalado anteriormente, entre las dos formas de justicia.

Traducción libre de José Ramiro LLatas Pérez, abogado especialista en Derechos Humanos.

42 BANKOWSKI e MACLEAN, Introduction, in The Universal, cit., p.xii. 
Bouvier, H. (2012) Particularismo y Derecho. Un abordaje postpositivista en el ámbito práctico. Marcial Pons, Ediciones Jurídicas y Sociales.

Calamandrei, P. (1920) La Cassazione civile.II.Disegno generale dell'istituto [La Casación Civil. II. Diseño general del instituto] (Volumen VII). Editora Fratelli Bocca.

Engisch, K. (1963). Logische Studien Zur Gesetzesanwendung. Katalog Für Die Bibliotheken Der Universität Heidelberg.

Gentili, A. (2013) Il diritto come discorso [La Ley como discurso]. Editorial Giuffre.

Grossi, P. (2011) Universalismo e particolarismo nel diritto [Universalismo y particularismo en el Derecho]. Editoriale Scientifica.

Higashiyama, E. (2011) Teoria do Direito Sumular [Teoría del Derecho Sumario]. Revista de Processo. 71-124.

Luzzati, C. (Agosto del 2013). Il giurista che cambia e non cambia. Diritto pubblico, 385-440. MacLean, J., \& Bankowski, Z. (Eds.) (2006). The Universal and the Particular in Legal Reasoning. Publicación Ashgate.

Maccormick, N. (2009). Particulars. DOXA, 127-150.

Nitsch, C. (2012). Il Giudice E La Legge. Consolidamento E Crisi Di Un Paradigma Nella Cultura Giuridica Italiana Del Primo Novecento. Editorial Dott. A. Giuffrè Editore S.p.a.

Palazzo, F. Il diritto penale tra universalismo e particolarismo [Derecho penal entre universalismo y particularismo]. Editoriale Scientifica.

Pavlakos, G. (Mayo del 2005). Two Conceptions of Universalisation. Red de Investigación en Ciencias Sociales.

Rudovkas, A. (2014) Precedente giudiziario come fonte del diritto nell'ordinamento giuridico della Federazione Russa, en Casuística y Jurisprudencia. 129 -137.

Sacco, R. (2010) Il diritto tra uniformazione e particolarismi [La ley entre uniformidad y particularidades]. Editoriale Scientifica.

Santos, A., Díez-Picazo, I., Vegas, J. (2012) Curso de Derecho Procesal Civil II. Parte especial. Universitaria Ramón Areces, Centro de Estudios Ramón Areces S. A.

Taruffo, M. (1991) Calamandrei e l'unificazione della Cassazione [Calamandrei y la Unificación del Tribunal Supremo].

Taruffo, M. (1994) Enciclopedia Treccani Scienze Sociali [Enciclopedia Treccani Ciencis Sociales] (Volumen IV)

Taruffo, M. (Enero del 2014) Il Fatto E L'interpretazione, In La Fabbrica Delle Interpretazioni. Càtedra de Cultura Jurídica, Girona.

Taruffo, M. (1991) La Corte di Cassazione e la legge [El Tribunal de Casación y la Ley].

Taruffo, M. (1992). La prova dei fatti giuridici. Nozioni generali. Editorial Giuffre.

Taruffo, M. (2009) La Semplice Verità. Editorial Lazerta.

Taruffo, M. (Diciembre del 2007). Precedente y Jurisprudencia. Precedente. Revista Jurídica. (-), 86-99. 